\title{
A Combined Approach for Face Detection
}

\author{
C.Victoria Priscilla \\ Assistant Professor, Information Technology, \\ S.D.N.B.Vaishnav College For women, Chennai.
}

\author{
B.Poorna \\ Professor and Principal, \\ SSS Shasun College for Women, Chennai
}

\begin{abstract}
This paper aims to detect the face in the digital image with the combination of morphological operations, Local Binary Pattern descriptor and with the moment invariants. In the digital image, the detection of face has gained much importance in the last decade, with enormous applications in the field of law enforcement and security. Although, to detect the face is an extremely simple task for the human eye, automating the process to a computer requires the use of various image processing techniques. In this paper since the combined approach is used, it strengthens the detection phase and also it helps to find the symmetric property of the face. Since the face detection is the initial step for face recognition, this proposed method can be used to detect the face in recognition of the face.
\end{abstract}

Keywords: face detection, morphological operations, Local Binary Pattern, Moment invariants.

\section{INTRODUCTION}

Face recognition [1] is a major area of research within biometric signal processing. A variety of approaches have been suggested for segmenting the human face from images [2],[3]. The localization of human faces in digital images is a fundamental step in the process of face recognition. An image of a face can be considered as a set of features such as eyes, mouth, nose with constrained positions and size within an oval: an explicit model can be used. One of the simplest and fastest methods to realize the feature extraction is the projection of the image or the edge image on the vertical axis to find the eyes or the mouth and on the horizontal axis to locate the nose [4]. The most significant problem in face detection and identification is to segment the human face from its background scene. Generally there are three phases [5] for human face segmentation and detection namely, faces representation, face segmentation and face identification.

There are many closely related problems of face detection. Face localization aims to determine the image position of a single face; this is a simplified detection problem with the assumption that an input image contains only one face [6], [7], [8]. The goal of facial feature detection [9], [10] is to detect the presence and location of features, such as eyes, nose, nostrils, eyebrow, mouth, lips, ears, etc., with the assumption that there is only one face in an image.

\subsection{Face detection Methods}

Existing techniques are available to detect faces from a single intensity or color image. There are many methods have been suggested to detect faces in a photo, but basically face detection methods [6],[11] can be classified in to four categories:

1. Knowledge-based methods. These rule-based methods encode human knowledge of what constitutes a typical face. Usually, the rules capture the relationships between facial features. These methods are designed mainly for face localization.

2. Feature invariant approaches. These algorithms aim to find structural features that exist even when the pose, viewpoint, or lighting conditions vary, and then use to locate faces. These methods are designed mainly for face localization.

3. Template matching methods. Several standard patterns of a face are stored to describe the face as a whole or the facial features separately. The correlations between an input image and the stored patterns are computed for detection. These methods have been used for both face localization and detection.

4. Appearance-based methods. In contrast to template matching, the models (or templates) are learned from a set of training images which should capture the representative variability of facial appearance. These learned models are then used for detection. These methods are designed mainly for face detection.

In this knowledge based approach, face detection methods are developed based on the rules derived from the researcher's knowledge of human faces. It is easy to come up with simple rules to describe the features of a face and their relationships. For example, a face often appears in an image with two eyes that are symmetric to each other, a nose, and a mouth. The relationships between features can be represented by their relative distances and positions.

\subsection{Local Binary Pattern}

During the past few years, local binary patterns (LBPs) [12] have aroused increasing interest in image processing and computer vision. As a nonparametric method, LBP summarizes local structures of images efficiently by comparing each pixel with its neighbouring pixels. LBP is one of the best texture methods available today. It is invariant to monotonic changes in gray-scale and fast to calculate. Its efficiency originates from the detection of different micro patterns (edges, points, constant areas etc.). LBP has already proved its worth in many applications [13], [14] in which texture plays an important role.

The LBP operator [15] was first introduced as a complementary measure for local image contrast. The LBP operator is one of the best performing texture descriptors and it has been widely used in various applications. The first incarnation of the operator worked with the eight-neighbours of a pixel, using the value of the center pixel as a threshold. An LBP code for a neighborhood was produced by multiplying the threshold values with weights given to the corresponding pixels, and summing up the result.

Recently, study of machine vision [16] tasks which have not been previously considered texture analysis problems. The LBP methodology has been adapted to outdoor scene classification, face recognition, face detection, facial 
expression recognition and content based image retrieval with excellent success. The LBP operator has been made into a really powerful measure of image texture, showing excellent results in terms of accuracy and computational complexity in many empirical studies. A novel approach to face recognition was developed which considers both shape and texture information to represent face images [13]. The face area is first divided into several small regions from which the LBP features are extracted and concatenated into an enhanced feature vector to be used as a face descriptor. The LBP operator was originally designed for texture description. The LBP [13] operator assigns a label to every pixel of an image by thresholding the $3 \times 3$-neighborhood of each pixel with the center pixel value and considering the result as a binary number. Then the histogram of the labels can be used as a texture descriptor.

\begin{tabular}{|c|c|c|}
\hline 5 & 9 & 1 \\
\hline 4 & 4 & 6 \\
\hline 7 & 2 & 3 \\
\hline
\end{tabular}$\stackrel{\text { Threshold }}{\longrightarrow}$\begin{tabular}{|c|c|c|}
\hline 1 & 1 & 0 \\
\hline 1 & & 1 \\
\hline 1 & 0 & 0 \\
\hline
\end{tabular} $\begin{aligned} & \text { Binary: } 11010011 \\
& \text { Decimal: } 211\end{aligned}$

Fig 1. Basic Local Binary Pattern

\subsection{Morphological operations}

Image processing techniques have been tremendously developed during the past five decades, and among them, mathematical morphology has been continuously receiving a great deal of attention. It is because mathematical morphology provides quantitative description of geometric structure and shape, as well as mathematical description of algebra, topology, probability, and integral geometry. Mathematical morphology [17] has been proved to be extremely useful in many image processing and analysis applications. Mathematical morphology can extract image shape features, such as edges, fillets, holes, corners, wedges, and cracks, by operating with various shaped structuring elements. In industrial vision applications mathematical morphology can be used to implement fast object recognition, image enhancement, segmentation, and defect inspection.

Morphology is the study of the form of things. Morphological transforms are designed to find this structure. Morphological transforms are usually applied to thresholded data, but can equally well be defined for greyscale and colour images. There are two fundamental transforms: erosion and dilation that can be combined in two ways to give two derived operations: opening and closing. Further combinations are defined to achieve other effects.

Morphological operations firstly require a structuring element. This is simply a kernel that defines a shape, commonly a circle, square or cross; others are equally possible and may be more useful in specific circumstances.

\subsubsection{Dilation and erosion}

Dilation and erosion [18] are basic morphological processing operations. They are defined in terms of more elementary set operations, but are employed as the basic elements of many algorithms. Both dilation and erosion are produced by the interaction of a set called a structuring element with a set of pixels of interest in the image. The structuring element has both a shape and an origin.

\subsubsection{Opening/Closing}

By utilizing the processes of erosion and dilation, opening and closing [18] is simply and extension of these applications. The process of "opening" an image will likely smooth the edges, break narrow block connectors and remove small protrusions from a reference image. "Closing" an image will also smooth edges but will fuse narrow blocks and fill in holes.

\subsection{Moment invariants}

Shape is known as one of the most important visual features, since it enables us to recognize objects without using further information. The approach using invariant features appears to be the most promising and has been used extensively. Its basic idea is to describe the objects by a set of measurable quantities called invariants that are insensitive to particular deformations and that provide enough discrimination power to distinguish among objects belonging to different classes. Consequently, shape representation, recognition and matching are considered to be an important element in image processing field. Moment invariants were firstly introduced to the pattern recognition community in 1962 by $\mathrm{Hu}$ [19], who employed the results of the theory of algebraic invariants and derived his seven famous invariants to rotation of 2-D objects. The idea of using moments in shape recognition gained prominence when $\mathrm{Hu}$ [19] derived a set of invariants using algebraic invariants. Shape representation methods focus either on shape boundaries (boundary-based methods) which use curvature [20] or on interior region of shape (region based methods) which use moments [21] [22]. It is possible to calculate moments [23] which are invariant under translation, changes in scale, and also rotation.

Most frequently used are the $\mathrm{Hu}[19]$ computed by normalizing central moments through order three, that are invariant to object scale, position, and orientation. In terms of the central moments, the seven moments are given as,

$$
\begin{aligned}
I_{1}= & \eta_{20}+\eta_{02} \\
I_{2}= & \left(\eta_{20}-\eta_{02}\right)^{2}+\left(2 \eta_{11}\right)^{2} \\
I_{3}= & \left(\eta_{30}-3 \eta_{12}\right)^{2}+\left(3 \eta_{21}-\eta_{03}\right)^{2} \\
I_{4}= & \left(\eta_{30}+\eta_{12}\right)^{2}+\left(\eta_{21}+\eta_{03}\right)^{2} \\
I_{5}= & \left(\eta_{30}-3 \eta_{12}\right)\left(\eta_{30}+\eta_{12}\right)\left[\left(\eta_{30}+\eta_{12}\right)^{2}-3\left(\eta_{21}+\eta_{03}\right)^{2}\right]+ \\
& \left(3 \eta_{21}-\eta_{03}\right)\left(\eta_{21}+\eta_{03}\right)\left[3\left(\eta_{30}+\eta_{12}\right)^{2}-\left(\eta_{21}+\eta_{03}\right)^{2}\right] \\
I_{6}= & \left(\eta_{20}-\eta_{02}\right)\left[\left(\eta_{30}+\eta_{12}\right)^{2}-\left(\eta_{21}+\eta_{03}\right)^{2}\right]+4 \eta_{11}\left(\eta_{30}+\eta_{12}\right)\left(\eta_{21}+\eta_{03}\right) \\
I_{7}= & \left(3 \eta_{21}-\eta_{03}\right)\left(\eta_{30}+\eta_{12}\right)\left[\left(\eta_{30}+\eta_{12}\right)^{2}-3\left(\eta_{21}+\eta_{03}\right)^{2}\right]- \\
& \left(\eta_{30}-3 \eta_{12}\right)\left(\eta_{21}+\eta_{03}\right)\left[3\left(\eta_{30}+\eta_{12}\right)^{2}-\left(\eta_{21}+\eta_{03}\right)^{2}\right] .
\end{aligned}
$$

\section{PROPOSED METHOD}

Face detection is an important role and the initial step towards face recognition. In this method, the face is detected and confirmed using morphological operations, Local binary pattern and moment invariants. Using the above technique the proposed method is able to detect the face and it can find that the detected face can be symmetric to each other. In the Local Binary Pattern a binary code is produced by thresholding its value with the value of the center pixel. Normally the histogram is created to collect up the occurrences of different 
binary patterns. In this method after texture analysis, Moment invariants are applied to determine the features of the image. The proposed method is categorized into 3 important steps: i) once the input image is acquired, after thresholding, the morphological operations are applied, perform Local Binary Pattern and find moments invariants. ii)Detect the edges, perform the Local Binary Pattern and find moments invariants. iii) finally, find the invariant moments for the original face. Based on the combination of these steps, the acquired image is confirmed as face or not face. If the face is detected, then it is also able to find that both the sides are symmetric to each other.

\section{ALGORITHM}

The algorithm is implemented with the following steps for different face images in the independent database and with the other images.

Step 1: input the image as in fig.1a.

Step 2: This luminance part is then transformed to binary image with some threshold.

Step 3: After thresholding, opening and closing operations are performed to remove noise using the morphological operations as in fig. $1 \mathrm{~b}$.

Step 4: Detect the edges using predefined edge detector as in fig 1c. Apply the binary pattern technique similar to the Local Binary Pattern to the same image.

Step 5: The edges in the image looks thickened and clear.
Step 6: find the centroid for the blob in the image. Based on the centroid divide the face vertically based on the line perpendicular to the centroid.

Step 7: The face can be divided as in fig $1 \mathrm{~d}$ and fig 1e. Find the invariant moments for the both the parts.

Step 8: Apply LBP and find the invariant moments for the right side and left side of the Original detected face.

Step 9: Find only the invariant moments for the right side and left side of the Original detected face.

Step 10: Compare the invariant moments for the step7, step8 and step 9 .

Step 11: Based on the threshold value and the Euclidean distance, face is detected and predicted that it is symmetric to each other.

\section{EXPERIMENTAL RESULTS AND DISCUSSION}

The algorithm is evaluated and tested with the Indian face database and with the number of faces acquired from the web. The proposed method is evaluated using different images with various dimensions. The system is able to detect the face and not face with the initial step of morphological operations and the texture and shape features are extracted with the Local Binary Pattern and Moment invariants. If the face is detected then it is able to determine it is symmetric to each other.

Fig 1a: Input Face

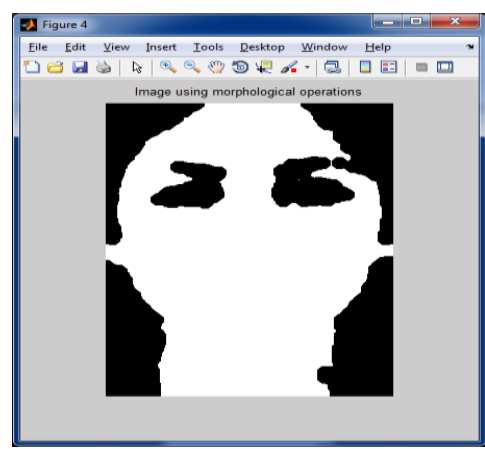

Fig 1b: dilated image

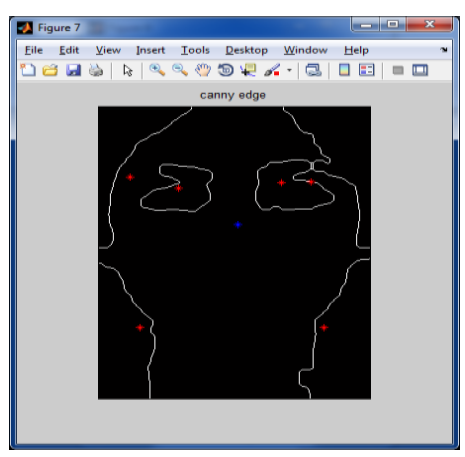

Fig 1c: Edge detection

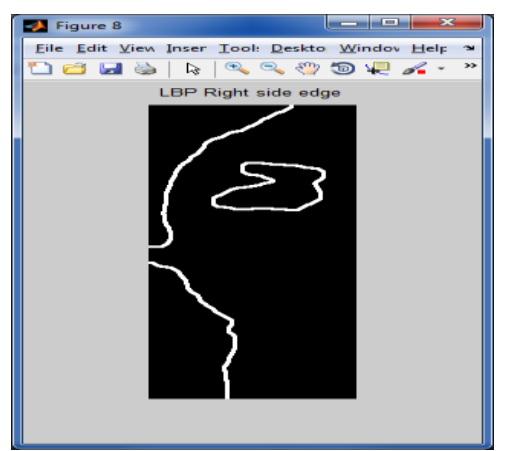

Fig 1d: Edge detection with LBP for

left side 

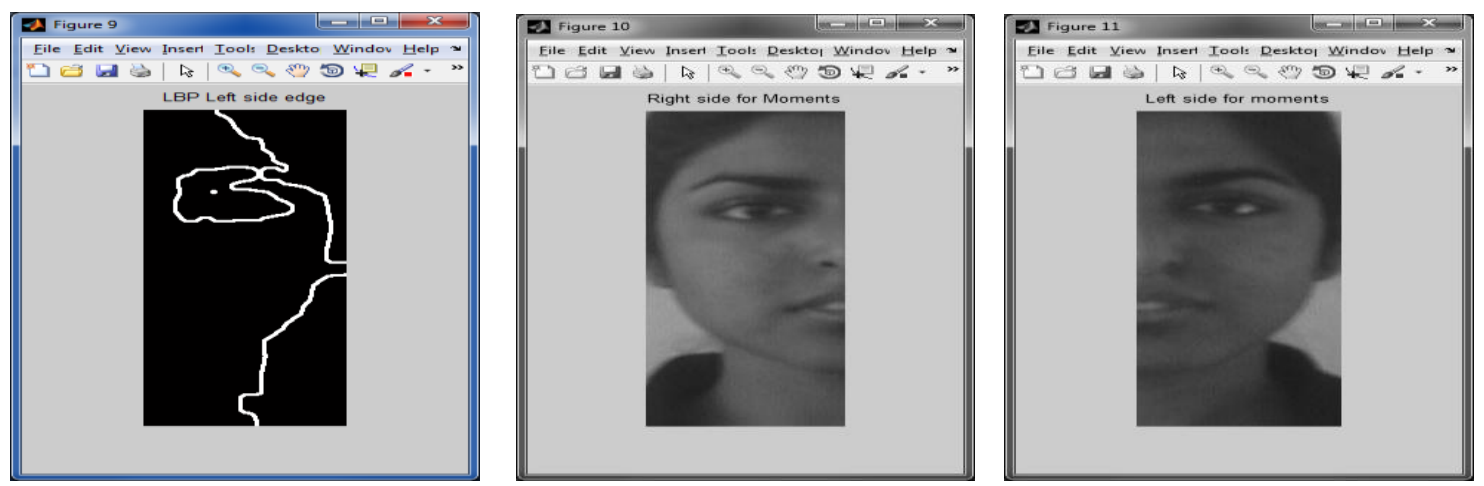

Fig 1e: Edge detection with LBP Fig 1f: Original face with Right side Fig 1g: Original face with Left side right side

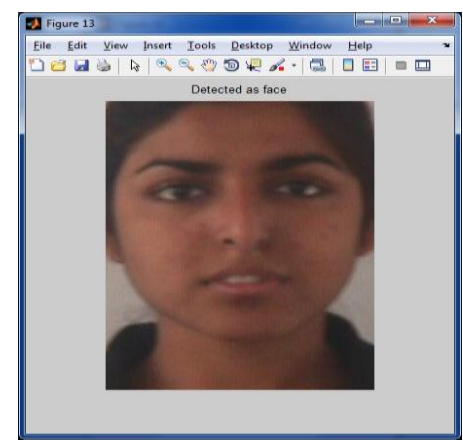

Fig 1h: Detected as face

Fig 1: Detected result for face

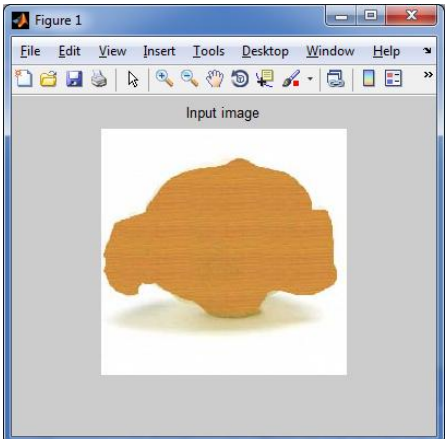

Fig 2a: Input Face

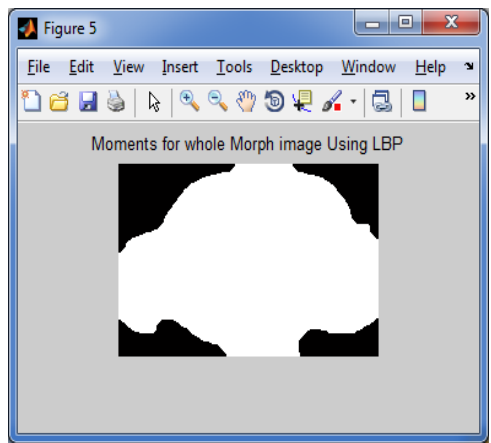

Fig 2b: Dilated image

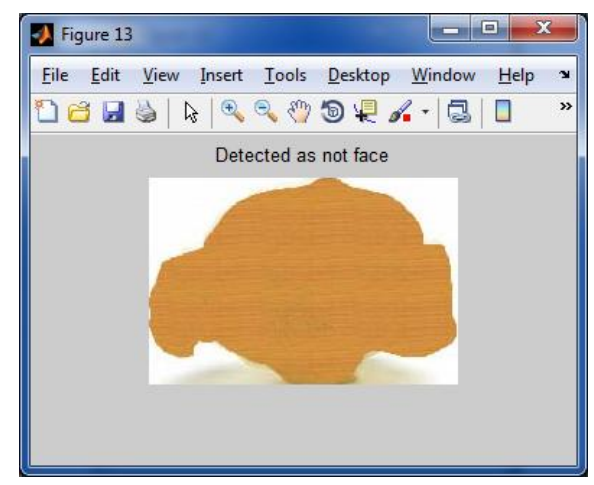

Fig 2c: Detected as not face

Fig 2: Detected result for Non face 
Table I. shows the moment invariants when the image is detected as face. It shows similarity of the values for the image using moment invariants. Based on these values and with thresholding, it is able to detect the face image as face and the other image which is not a face image is detected as not a face. Table II shows the detection evaluation results based on the proposed method which shows the four possible classifications, a true positive, a true negative, a false positive, a false negative. In order to evaluate the performance of this proposed method the performance metrics include precision and recall as shown in Table III were used. Other performance metrics [24] include precision and recall defined as follows: In the field of information retrieval, precision is the fraction of retrieved documents that are relevant to the search:

\section{Precision $=$ TP $/$ TP + FP}

Recall in information retrieval is the fraction of the documents that are relevant to the query that are successfully retrieved.

Recall $=\mathbf{T P} / \mathbf{T P}+\mathbf{F N}$

A number of model performance metrics can be derived from the confusion matrix. The most common metric is accuracy defined by

Accuracy $=$ TP + TN $/$ TP + TN + FP+FN

Table 1. Measures of moment invariants for face

\begin{tabular}{|c|c|c|c|c|c|c|}
\hline \multirow[t]{2}{*}{$\begin{array}{l}\text { Invariant } \\
\text { moments }\end{array}$} & \multicolumn{2}{|c|}{$\begin{array}{l}\text { Moments for both sides of } \\
\text { the face }\end{array}$} & \multicolumn{2}{|c|}{$\begin{array}{l}\text { Moments after LBP for both side } \\
\text { of the face }\end{array}$} & \multicolumn{2}{|c|}{$\begin{array}{l}\text { LBP and moments for face with } \\
\text { morphological operations }\end{array}$} \\
\hline & Left side & Right side & Left side & Right side & Left side & Right side \\
\hline $\begin{array}{l}\text { M5 } \\
\text { M6 } \\
\text { M7 }\end{array}$ & $\begin{array}{l}48.3891 \\
33.0654 \\
47.4312\end{array}$ & $\begin{array}{l}5.7496 \\
12.1915 \\
21.2860 \\
22.6015 \\
44.7842 \\
29.4666 \\
45.0291\end{array}$ & $\begin{array}{l}6.2957 \\
13.1297 \\
24.5394 \\
24.2875 \\
49.0699 \\
30.8751 \\
50.8109\end{array}$ & $\begin{array}{l}6.3256 \\
13.2023 \\
23.6382 \\
23.8084 \\
47.0944 \\
30.4793 \\
51.8781\end{array}$ & $\begin{array}{l}6.6926 \\
13.8574 \\
22.9544 \\
25.4640 \\
49.4677 \\
32.7037 \\
50.1049\end{array}$ & $\begin{array}{l}6.6616 \\
13.8054 \\
22.3984 \\
24.1829 \\
47.2837 \\
33.3674 \\
47.7008\end{array}$ \\
\hline
\end{tabular}

Table II. Detection Evaluation results based on proposed method

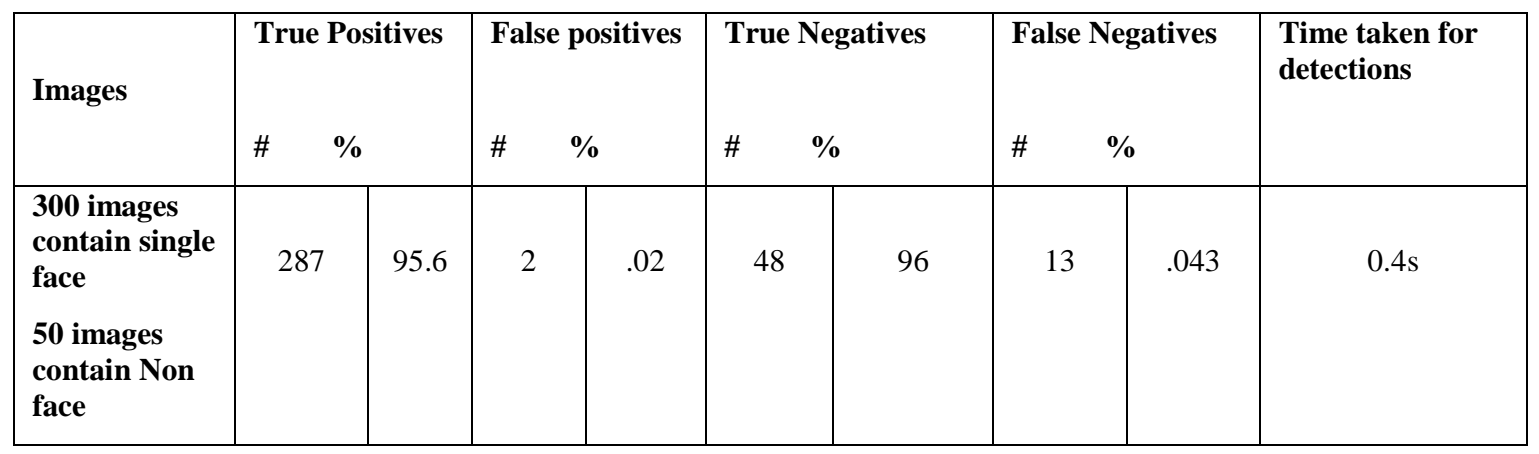

Table III. Performance Evaluation results

\begin{tabular}{|l|c|c|c|c|}
\hline Images & Number of images & Recall rate & Precision rate & Accuracy \\
\hline $\begin{array}{l}\text { images contain face } \\
\text { and Non face }\end{array}$ & $300+50$ & 0.9566 & 0.993 & 0.9571 \\
\hline
\end{tabular}




\section{COMPARISONS OF EXPERIMENTAL RESULTS BY VARIOUS METHODS}

The main aim of the proposed face detection algorithm is to combine the various techniques to detect the face in the given image and to reduce the false positive rates which help in recognition of the face. The following Table IV shows the comparison results of the various methods, and the proposed method shows the better accuracy compared with other methods to detect the face and the fig 3. is the histogram which explains the comparison between the proposed method and previous methods. From this histogram, we can see our proposed method has the highest and the detection rate of $95.71 \%$

Table IV. Comparison of experimental results

\begin{tabular}{|c|l|c|}
\hline S.No & $\begin{array}{l}\text { Various Methods for face } \\
\text { detection }\end{array}$ & $\begin{array}{l}\text { Detection } \\
\text { rate/success rate } \\
\text { in \% }\end{array}$ \\
\hline 1 & $\begin{array}{l}\text { Morphological operations } \\
\text { with classifier [25] } \\
\text { (MOC) }\end{array}$ & $94 \%$ \\
\hline 2 & $\begin{array}{l}\text { Morphological operations } \\
\text { with color based } \\
\text { segmentation [26] (MOS) }\end{array}$ & $90 \%$ \\
\hline 3 & $\begin{array}{l}\text { Moment invariants and skin } \\
\text { color model with classifier } \\
{[27](\text { MISC) }}\end{array}$ & $85 \%$ \\
\hline 4 & $\begin{array}{l}\text { Proposed method } \\
\text { (Morphological operations }+ \\
\text { Local Binary Pattern }+ \\
\text { Moment inavriants) }\end{array}$ & \\
\hline
\end{tabular}

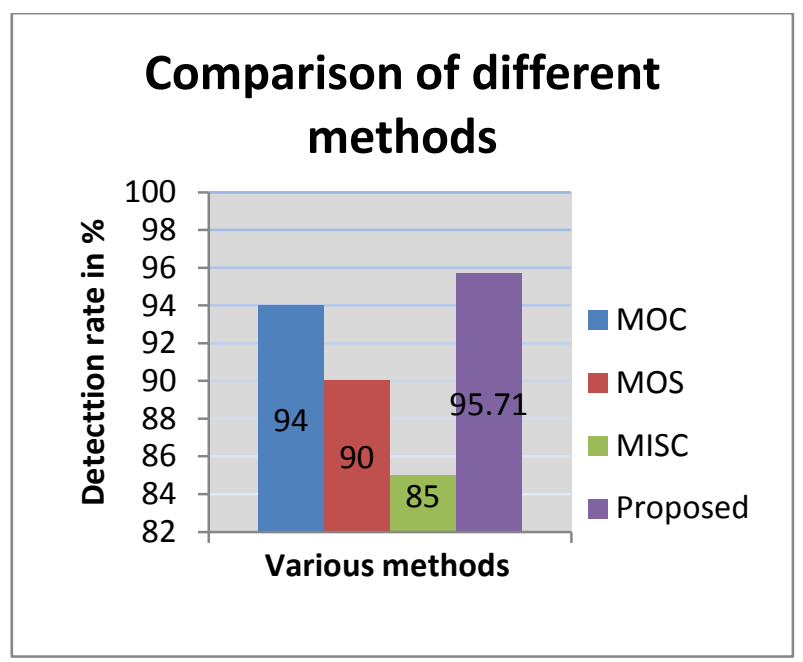

Fig.3 Comparison between proposed method and previous methods

\section{CONCLUSION}

The proposed method is very simple and is able to detect the face with frontal views. The key idea is to detect the face and the non face. Even though the face is difficult to detect, since the shape and the texture characteristics are extracted as features using Local Binary Pattern and the moment invariants it strengthens the detecting phase. This method can be used in recognition of the face. Thus the average accuracy of the proposed method is $95 \%$. In future research, the complicated pose variations; side face can be included for the same. This method can extend in future to determine the human face in the complex background with more datasets.

\section{REFERENCES}

[1]. Oliver Jesorsky, Klaus J. Kirchberg, and Robert W. Frischholz, "Robust Face Detecion Using the Hausdorff Distance", In Proc. Third International Conference on Audio- and Video-based Biometric Person Authentication, Springer, Lecture Notes in Computer Science, LNCS-2091, pp. 90-95, Halmstad, Sweden, 6-8 June 2001.

[2]. R. Chellappa, C.L. Wilson, S. Sirohey, "Human and machine recognition of faces: A survey", Proc. of IEEE, V83, 1995, pp. 705-740.

[3]. A. Samal, P. A. Iyengar "Automatic recognition and analysis of human faces and facial expressions: A survey”, Pattern Recognition, 25(1), 1992, pp. 65-77.

[4]. Raphael Feraud, Olivier J. Bernier, Jean-Emmanuel Viallet, and Michel Collobert, "A Fast and Accurate Face Detector Based on Neural Networks", IEEE TRANSACTIONS ON PATTERN ANALYSIS AND MACHINE INTELLIGENCE, VOL. 23, NO. 1, JANUARY 2001

[5]. J. Prakash, and K. Rajesh, "Human Face Detection and Segmentation using Eigenvalues of Covariance Matrix, Hough Transform and Raster Scan Algorithm", World Academy of Science, Engineering and Technology 39 ,2008.

[6]. Emad Nikkhouy and Eimad Eldin Abdu Abusham, "Facial features detection using Eyes-Nose template", IJCSNS International Journal of Computer Science and Network Security, VOL.11 No.11, November 2011.

[7]. K. Lam and H. Yan, "Fast Algorithm for Locating Head Boundaries," J. Electronic Imaging, vol. 3, no. 4, pp. 351-359, 1994

[8]. B. Moghaddam and A. Pentland, "Probabilistic Visual Learning for Object Recognition," IEEE Trans. Pattern Analysis and Machine Intelligence, vol. 19, no. 7, pp. 696-710, July 1997.

[9]. I. Craw, D. Tock, and A. Bennett, "Finding Face Features," Proc. Second European Conf. Computer Vision, pp. 92-96, 1992.

[10]. H.P. Graf, T. Chen, E. Petajan, and E. Cosatto, "Locating Faces and Facial Parts," Proc. First Int'l Workshop Automatic Face and Gesture Recognition, pp. 41-46, 1995.

[11].Ming-Hsuan Yang, David J. Kriegman, and Narendra Ahuja, " Detecting Faces in Images: A Survey", IEEE TRANSACTIONS ON PATTERN ANALYSIS AND MACHINE INTELLIGENCE, VOL. 24, NO. 1, JANUARY 2002.

[12].Di Huang, Caifeng Shan, Mohsen Ardabilian, "Local Binary Patterns and Its Application to Facial Image Analysis: A Survey", IEEE TRANSACTIONS ON SYSTEMS, MAN, AND CYBERNETICS-PART C: 
APPLICATIONS AND REVIEWS, VOL. 41, NO. 6, NOVEMBER 2011.

[13]. Ahonen T, Hadid A, Pietikainen M “ Face Recognition with Local Binary Patterns" Lecture Notes in Computer Science, Vol. 3021. Springer-Verlag, Berlin Heidelberg New York (2004) 469-481.

[14]. Valtteri Takala, Timo Ahonen, and Matti Pietikainen,"Block-Based Methods for Image Retrieval Using Local Binary Patterns",H. Kalviainen et al. (Eds.): SCIA 2005, LNCS 3540, pp. 882-891, 2005.SpringerVerlag Berlin Heidelberg 2005

[15].T. Ojala, M. Pietikainen, and D. Harwood, "A comparative study of texture measures with classification based on feature distributions," Pattern Recognition, vol. 29, no. 1, pp. 51-59,1996.

[16].H. Kalviainen et al., ".Image Analysis with Local Binary Patterns", (Eds.): SCIA 2005, LNCS 3540, pp. $115-$ 118, 2005 Matti Pietikäinen,Springer-Verlag Berlin Heidelberg 2005.

[17].Maragos, P. and Schafer, R., "Morphological filters. Part II: their relations to median, order-statistics, and stack filters," IEEE Trans. Acoust. Speech Signal Process., vol. 35, no. 8, pp. 1170-1184, Aug. 1987b.

[18].R. C. Gonzalez and R. E.Woods, "Digital Image Processing”. PrenticeHall, 3rd ed., 2007.

[19].M. K. Hu, "Visual pattern recognition by moment invariants," IRE Trans. Information Theory, vol. 8, pp. $179-187,1962$
[20].F. Mokhtarian and A. Sadegh, "Shape similarity retrieval under affine transforms," Pattern Recognition, no. 35, pp. $31-41,2002$

[21]. A. Zhao and J. Chen, "Affine curve moment invariants for shape recognition," Pattern Recognition, vol. 6, no. 30, pp. 895-901, 1997.

[22]. J. Flusser and T. Suk, "Pattern recognition by affine moment invariants," Pattern Recognition, vol. 1, pp. 167-174, 1993.

[23]. http://en.wikipedia.org/wiki/Image_moment

[24].http://en.wikipedia.org/wiki/Accuracy

[25].Chin-Chuan Han, Hong-Yuan Mark Liao, Gwo-Jong Yu, Liang-Hua Chen, "Fast face detection via morphologybased pre-processing", Pattern Recognition 33 (2000) $1701\} 1712$

[26].Arti Khaparde, Sowmya Reddy.Y Swetha Ravipudi, "Face Detection Using Color Based Segmentation and Morphological Processing -A Case Study", Proceedings of ISCET 2010, ISBN : 978-81-910304-0-2

[27]. Jean-Christophe Terrillon, Martin David and Shigeru Akamatsu ,Automatic Detection of Human Faces in Natural Scene Images by Use of a Skin Color Model and of Invariant Moments, Proceedings of Third IEEE International Conference on Automatic Face and Gesture Recognition FG'98,1998 